\title{
TOWARDS A MATROID-MINOR STRUCTURE THEORY
}

\author{
JIM GEELEN, BERT GERARDS, AND GEOFF WHITTLE
}

\begin{abstract}
This paper surveys recent work that is aimed at generalising the results and techniques of the Graph Minors Project of Robertson and Seymour to matroids.
\end{abstract}

For Dominic Welsh, on the occasion of his retirement.

\section{INTRODUCTION}

A number of the most interesting and apparently difficult conjectures in matroid theory concern minor-closed classes. We begin by describing three fundamental conjectures of this type.

Perhaps the most famous such conjecture is Rota's Conjecture [27]. A minor-minimal matroid that does not belong to a given minor-closed class of matroids is an excluded minor for that class.

Conjecture 1.1 (Rota's Conjecture). Let $\mathbb{F}$ be a finite field. There are, up to isomorphism, only finitely many excluded minors for the class of $\mathbb{F}$ representable matroids.

Lazarson [19] showed that there are an infinite number of excluded minors for representability over the reals and this is certainly true for all other infinite fields, so, if true, Rota's Conjecture is best possible.

Tutte [29] proved that a matroid is binary if and only if it has no $U_{2,4^{-}}$ minor. In $[1,28]$ it is proved that a matroid is ternary if and only if it has no $U_{2,5}, U_{3,5}, F_{7}$ or $F_{7}^{*}$-minor. No real progress was made for twenty years until Geelen, Gerards and Kapoor [5] proved Rota's Conjecture for $G F(4)$ showing that there were seven excluded minors for $G F(4)$-representability.

Other fundamental conjectures are inspired by the groundbreaking work of Robertson and Seymour in their celebrated Graph Minors Project. A major outcome of this project is their proof of Wagner's Conjecture establishing that graphs are well-quasi-ordered under the minor order [25]. In other words, in any infinite sequence of graphs there is one that is isomorphic to a minor of another. The conjectured extension to matroids was certainly made by Robertson and Seymour, although apparently not in print.

Conjecture 1.2 (Well-Quasi-Ordering Conjecture). Let $\mathbb{F}$ be a finite field. Then any infinite set of $\mathbb{F}$-representable matroids contains two matroids, one of which is isomorphic to a minor of the other.

1991 Mathematics Subject Classification. 05B35. 
As yet, the Well-Quasi-Ordering Conjecture has not been resolved for any finite field. Note that the Well-Quasi-Ordering Conjecture is equivalent to the conjecture that for a finite field $\mathbb{F}$, any minor closed class of $\mathbb{F}$-representable matroids has a finite number of $\mathbb{F}$-representable excluded minors.

The Well-Quasi-Ordering Conjecture is best possible in the sense that it is easy to construct infinte antichains of matroids that are representable over any infinite field. Here is an elementary one. For an integer $n \geq 3$, let $P_{n}$ be the matroid on $\left\{p_{1}, \ldots, p_{n}, q_{1}, \ldots, q_{n}\right\}$ where $\left\{p_{1}, \ldots, p_{n}\right\}$ are the vertices of a regular $n$-gon in the affine plane and place each point $q_{i}$ freely on the line spanned by $\left\{p_{i}, p_{i+1}\right\}$ in the circular order. Then it is routinely verified that $\left\{P_{3}, P_{4}, \ldots\right\}$ is an infinite antichain of rank-3 matroids. It is also easily seen that each $P_{n}$ is representable over any infinite field.

The Graph Minors Project also has fundamental algorithmic consequences. In particular Robertson and Seymour [24] prove that there is a polynomial-time algorithm for recognising a given graph as a minor. As a consequence any minor-closed property of graphs can be recognised in polynomial time. We conjecture that this result extends to matroids representable over finite fields.

Conjecture 1.3 (Minor-Recognition Conjecture). For any finite field $\mathbb{F}$ and $\mathbb{F}$-representable matroid $N$, there is a polynomial-time algorithm for testing whether an $\mathbb{F}$-representable matroid contains an $N$-minor.

Combined with the WQO Conjecture, Conjecture 1.3 implies that, for a given finite field $\mathbb{F}$, there is a polynomial-time algorithm for testing any minor-closed property for $\mathbb{F}$-representable matroids.

We are currenly undertaking a program of research aimed at extending the techniques of the Graph Minors Project to matroids with the eventual goal of resolving conjectures such as the ones described above.

At the heart of the Graph Minors Project is the Graph Minors Structure Theorem. This theorem provides a constructive characterisation for members of the class of graphs that do not contain a given graph as a minor. This structural characterisation is the workhorse of the Graph Minors Project. Having such a characterisation enables techniques to be brought to bear to establish well-quasi-ordering and algorithmic consequences.

Our hope is to use the same strategy for matroids. Indeed, this strategy has already had some success. It turns out that excluding a planar graph as a minor imposes tangible structure on a class of representable matroids, and we begin by discussing this.

\section{Excluding a Planar Graph}

Let $\mathbb{F}$ be a finite field and let $H$ be a planar graph. In this section we give a constructive structural description of $\mathbb{F}$-representable matroids with no $M(H)$-minor and show that this description enables significant progress on Conjectures 1.1, 1.2, and 1.1. 
Tree width is now a well understood parameter for graphs and we will not define it here. Intuitively a graph has low tree width if it can be obtained from a set of small graphs using clique-sums, or, put another way, it admits a tree-like decomposition into small pieces. A class of graphs has bounded tree width if there is an integer $k$ such that all members of the class have tree width at most $k$.

In [22] Robertson and Seymour prove:

Theorem 2.1. For any planar graph $H$, there is a $k$ such that if $G$ is a graph of tree width at least $k$, then $G$ has an $H$-minor.

Note that every planar graph is a minor of a grid; therefore it suffices to prove Theorem 2.1 for the case that $H$ is a grid. For this reason, Theorem 2.1 is often referred to as the Grid Theorem.

Let $\mathcal{G}$ be a class of graphs that does not have a fixed planar graph $H$ as a minor. By the Grid Theorem, $\mathcal{G}$ has bounded tree width, providing a constructive structural characterisation for members of the class. These structural properties provide considerable traction for both algorithmic and theoretical problems. For example, Robertson and Seymour [21] prove that any class of graphs of bounded tree width is well-quasi-ordered so that $\mathcal{G}$ is well-quasi-ordered.

While tree width does extend to matroids [17], the related notion of "branch width", introduced for graphs in [23], extends more naturally and is easier to work with. Branch width is equivalent to tree width in that a class of graphs, or matroids, has bounded branch width if and only if it has bounded tree width. Johnson, Robertson and Seymour [18] conjectured that Theorem 2.1 extends to all finite fields and this extension is achieved in $[12]$.

Theorem 2.2 (Grid Theorem for matroids). Let $H$ be a planar graph and $q$ be a prime power. Then there exists an integer $\omega(H, q)$ such that, if $M$ is a $G F(q)$-representable matroid with branch width at least $\omega(H, q)$, then $M$ has an $M(H)$-minor.

This theorem is absolutely central to our project, and was not easily achieved. There are at least three proofs of the Grid Theorem for graphs in the literature $[22,26,3]$. It is natural to attempt to extend the techniques in these proofs. In this respect [3] was particularly tantalizing, since it is the easiest of the proofs and the hard part of the proof has a matroidal flavour. Johnson, Robertson, and Seymour succeeded in generalizing the "hard part" to matroids, but, unfortunately, the "easy part" relied on properties that appear to be particular to graphs. When specialized to graphs, our proof of Theorem 2.2 is different from the existing proofs. It is important to note that we had access to an extraordinary 150 page handwritten manuscript of Johnson, Robertson, and Seymour describing their progress towards a grid theorem for matroids. The techniques we learned from their manuscript 
played a crucial role in our proof. The proof also makes use of earlier results we obtained together with Neil Robertson $[6,7]$.

It follows that any minor-closed class of $G F(q)$-representable matroids that does not contain all planar graphs has bounded branch width. In [8] it is proved that any class of $G F(q)$-representable matroids of bounded branch width is well-quasi-ordered under the minor order. As a consequence we obtain the following partial result towards the WQO-Conjecture.

Theorem 2.3. Let $\mathbb{F}$ be a finite field and $\mathcal{M}$ be a minor-closed class of $\mathbb{F}$ representable matroids that does not contain the cycle matroids of all planar graphs. Then $\mathcal{M}$ is well-quasi-ordered under the minor order.

In combination with results of Hliněný [16], we also obtain partial progress towards the Minor-Recognition Conjecture.

Theorem 2.4. For any finite field $\mathbb{F}$ and planar graph $H$, there is a polynomial-time algorithm for testing whether or not an $\mathbb{F}$-representable matroid contains an $M(H)$-minor.

In [14] it is shown that for a finite field $\mathbb{F}$ and integer $k$, there is a finite number of excluded minors for $\mathbb{F}$-representability of branch width at most $k$. In combination with the grid theorem this yields the following theorem.

Theorem 2.5. For any finite field $\mathbb{F}$ and planar graph $H$, there are a finite number of excluded minors for $\mathbb{F}$-representability that do not have $M(H)$ as a minor.

We see that the structure imposed on a class of matroids by excluding the matroid of a planar graph as a minor yields restricted solutions to Rota's Conjecture, the WQO Conjecture and the Minor Recognition Conjecture, and that is a promising beginning.

\section{Global and local structure}

The Graph Minors Structure Theorem provides a constructive structural description of the members of the class of graphs obtained by excluding an arbitrary graph $H$ as a minor. Intuitively, the theorem states that: any graph with no $H$-minor admits a tree-like decomposition into parts that essentially embed into some surface that $H$ does not. For applications, we typically consider the case that $H$ is a clique. The tree-decomposition provides "global structure", and the structure of the parts is referred to as "local structure". In Graph Minos X [23], Robertson and Seymour show that all graphs naturally exhibit tree-like decompositions into parts that are highly connected. This decomposition is obtained by considering the maximal "tangles" in a graph.

A separation of a graph $G$ is a pair $\left(G_{1}, G_{2}\right)$ of subgraphs of $G$ such that $G=G_{1} \cup G_{2}$; the order of the separation $\left(G_{1}, G_{2}\right)$ is $\left|V\left(G_{1}\right) \cap V\left(G_{2}\right)\right|$. A separation of a matroid $M$ is a partition $(A, B)$ of $E(M)$. The order of the separation $(A, B)$ is $r_{M}(A)+r_{M}(B)-r(M)+1$; for a representable matroid 
this is the dimension of the intersection of the subspaces spanned by $A$ and $B$ plus 1. Moreover, if $\left(G_{1}, G_{2}\right)$ is a separation of $G$ and $G_{1}, G_{2}$, and $G$ are connected, then the order of $\left(G_{1}, G_{2}\right)$ is equal to the order of the separation $\left(E\left(G_{1}\right), E\left(G_{2}\right)\right)$ in $M(G)$.

Just as one might call the blocks of a graph its "2-connected components", the tangles of order $k$ are its " $k$-connected components". To define a tangle of order $k$ we consider all separations $\left(G_{1}, G_{2}\right)$ of order $<k$, and assign one of $G_{1}$ and $G_{2}$ to be the small side in such a way that no three small sides cover the graph. Moreover, to avoid trivialities, no small side may be spanning. The definition for matroids is essentially the same.

Robertson and Seymour [23] prove that, each graph admits a tree-like decomposition whose parts are the maximal tangles. In [10] we generalize this to matroids: each matroid admits a tree-like decomposition whose parts are the maximal tangles. Now, to obtain a structural theorems it suffices to describe the structure of a graph or matroid local to each of its tangles. Generalizing results from [26], in [10] we proved that, for any planar graph $H$ and finite field $\mathbb{F}$, each tangle of sufficiently large order in an $\mathbb{F}$-representable matroid "controls" an $M(H)$-minor. (For the tangle to control a minor $N$, we mean that $E(N)$ is not contained in the small side of a separation of low order.) We typically choose $H$ to be a grid. This highly structured minor helps to get a handle on the local structure of the tangle.

Robertson and Seymour [23] also prove that, the maximum order of a tangle in a graph is equal to its branch width. Dhamatilike [2] extended this to matroids (although this result was implicit in [23]).

The above results provide a good handle on the global structure. We now turn to local structure, and give a clearer explanation of what we mean by this. Consider a separation $\left(G_{1}, G_{2}\right)$ in a graph $G$. We say that $H$ is obtained by reducing $G_{1}$ in $G$, if $H$ is obtained from $G_{2}$ by putting a clique on the vertex set $V\left(G_{1}\right) \cap V\left(G_{2}\right)$. Now, let $\mathcal{C}$ be a class of graphs and let $G$ be a graph with a given tangle $\mathcal{T}$. We say that $\mathcal{T}$ has local structure in $\mathcal{C}$, if there exist separations $\left(L_{1}, R_{1}\right), \ldots,\left(L_{k}, R_{k}\right)$ in $G$ with edge-disjoint small sides $L_{1}, \ldots, L_{k}$ such that the graph obtained from $G$ by reducing each of $L_{1}, \ldots, L_{k}$ is in $\mathcal{C}$.

To make the analogous definition for matroids, we need only describe what we mean by "reducing". Consider a separation $(A, B)$ in an $\mathbb{F}$-represented matroid $M$. To avoid trivial technicalities, suppose that $M$ is simple and is given as a restriction of a projective geometry. Let $C$ be the set of points in the projective geometry that are in the closure of $B$ and that are spanned by a set $A^{\prime} \subseteq A$ where $r_{M}\left(A^{\prime}\right)+r_{M}(B)=r_{M}\left(A^{\prime} \cup B\right)+1$. That is, $C$ is the set of points in the flat spanned by $B$ onto which we can contract elements of $A$. The matroid $N$ on $C \cup B$ is the matroid obtained from $M$ by reducing A. 


\section{Some Minor-Closed Classes}

Consider a finite field $\mathbb{F}$. In this section we develop natural minor-closed classes of $\mathbb{F}$-representable matroids in an attempt to describe the local structure of tangles.

Let us first consider minor-closed classes of graphs. For any surface $\Sigma$, the class of graphs that embed in $\Sigma$ is minor closed. Readers familiar with the Graph Minors Structure Theorem will also be aware that richer minorclosed classes can be obtained by adding a bounded number of structures called vortices to graphs embedded in $\Sigma$. Vortices are essentially graphs of bounded path width glued, in a particular way, to a face-boundary of a graph drawn in $\Sigma$. We can further embellish the class by adding vertices; given any minor-closed class $\mathcal{C}$ of graphs, we can construct a new minorclosed class by "apexing". That is, we construct all graphs that can be obtained by adding a new vertex $v$ to each graph in $\mathcal{C}$ and an arbitrary set of edges incident with $v$. The Graph Minors Structure Theorem shows that, in combination, these ideas suffice in describing the local structure of tangle in a graph with no $K_{n}$-minor.

What then might we expect in the class of $\mathbb{F}$-representable matroids? One natural minor-closed class is the class of graphic matroids. Also, if $\mathbb{F}^{\prime}$ is a subfield of $\mathbb{F}$, then the class of $\mathbb{F}^{\prime}$-representable matroids is a minorclosed class of $\mathbb{F}$-representable matroids. There is another natural class, generalizing the class of graphic matroids, that was originally introduced by Dowling [4], and then studied in greater depth by Zaslavsky [32]. We give a superficial treatment of the class here; for a guide to the extensive literature in the area see [33].

Dowling Matroids. Let $M$ be a matroid having a representation over a field $\mathbb{F}$ by a $V \times E(M)$ matrix $A$ with the property that every column of $A$ has at most two non-zero elements. The fact that $M$ has such a representation shows that $M$ is a Dowling matroid, and the representation $A$ is a Dowling representation. It is straightforward to prove that the class of Dowling matroids is minor closed. There are, in fact, other rich minor-closed classes associated with Dowling matroids.

Dowling Matroids and subgroups. Consider a Dowling representation $A$ of a simple matroid $M$. An element of $M$ that is represented by a column with a single nonzero element is a joint. (Note that the property of being a joint depends on the representation $A$.) Consider a non-joint element $e$ of $M$. By scaling we may assume that at least one of the two non-zero elements in the associated column is a 1 ; let $-\gamma_{e}$ denote the other nonzero element. Let $\Gamma$ be a subgroup of the multiplicative group $\mathbb{F}^{*}$ of $\mathbb{F}$. It is straightforward to verify that, if $\gamma_{e} \in \Gamma$ for each non-joint element of $M$, then each minor of $M$ has a Dowling representation with this same property. So we get a rich minor-closed class of $\mathbb{F}$ representable matroids from each subgroup of $\mathbb{F}^{*}$. 
Dowling matroids on surfaces. Consider a $V \times E(M)$ Dowling representation $A$ of a simple $\mathbb{F}$-representable matroid $M$. One can naturally associate a graph $G$ with $A$, where $V(G)=V$ and $E(G)$ is the set of nonjoint elements of $M$. If this graph embeds on a surface $\Sigma$, then each minor of $M$ has a Dowling representation whose associated graph also embeds on $\Sigma$. Thus, for each surface $\Sigma$ we obtain a rich minor-closed class of Dowling matroids. Moreover, in addition to the surface $\Sigma$ we can also specify a subgroup $\Gamma$ of $\mathbb{F}^{*}$ and consider Dowling matroids over $\Gamma$ embedded on $\Sigma$. In fact, we can further embellish this minor-closed class by allowing a bounded number of "vortices" (these are obtained by adding matroid elements into bounded-rank subspaces arranged in a cyclic manner around a face in the embedding).

We believe that the above classes are the building blocks for all minorclosed classes of $\mathbb{F}$-representable matroids. Each of these classes can be further embellished by "lifting" and "projecting", just as minor-closed classes of graphs can be embellished via apexing.

Projection. Projection is an "elementary" strong map. Let $M(A)$ be an $\mathbb{F}$-represented matroid. Now consider an extension $M([A, v])$ of $M(A)$ by an element $e$. Then, $M([A, v]) / e$ is a projection of $M(A)$. If $N$ is a projection of $M$, then we say that $N$ is obtained from $M$ by projecting or that $M$ is obtained from $N$ by lifting. Given any minor-closed class of $\mathbb{F}$-representable matroids, we can obtain a new minor-closed class by lifting and projecting a bounded number of times.

The operations of lifting and projecting can alternatively be viewed as "low-rank perturbations" of representations. We call a matrix $B$ a rank-k perturbation of a matrix $A$ if, after possibly appending zero-rows to $A$, we have $\operatorname{rank}(A-B)=k$. Similarly, we call $M(B)$ a rank- $k$ perturbation of $M(A)$ when $B$ is a rank- $k$ perturbation of $A$.

We complete this section by briefly returning to graphs. Consider a graph $G$ that is obtained by adding $k$ additional vertices, with arbitrary incident edges, to a graph embedded in a surface $\Sigma$. Let $G^{\prime}$ be obtained from $G$ by shrinking these $k$ new vertices to a single vertex $v$. Alternatively, we could have added $k-1$ edges to $G$ and then contracted them. Thus $M\left(G^{\prime}\right)$ is obtained from $M(G)$ by projecting $k-1$ times. Now let $A$ be the $V\left(G^{\prime}\right) \times$ $E\left(G^{\prime}\right)$ incidence matrix of $G^{\prime}$ and let $A^{\prime}$ be obtained from $A$ by deleting row $v$. Note that $M\left(G^{\prime}\right)=M\left(A^{\prime}\right)$ (considered over $\mathrm{GF}(2)$ ). Moreover, $A^{\prime}$ is a Dowling representation of $M\left(G^{\prime}\right)$ and the graph associated with this representation is $G^{\prime}-v$, which embeds in $\Sigma$. Thus the Graph Minors Structure Theorem is captured by the matroid classes given above.

\section{The local structure of a tangle}

In this section we state our main results and conjectures on the structure of minor-closed classes. Let $\mathbb{F}$ be a finite field of order $q=p^{k}$, where $p$ is prime and let $n$ be a positive integer. In each of these conjectures, $\mathcal{T}$ is a 
tangle in an $\mathbb{F}$-representable matroid $M$. Each of the implicit bounds given below depend only on $q$ and $n$, and not on $\mathcal{T}$ or $M$.

Excluding $M\left(K_{n}\right)$ and $M\left(K_{n}\right)^{*}$. Let $\mathcal{C}$ be the class of $\mathbb{F}$-representable matroids obtained in the following way. Take a Dowling matroid whose associated graph is embedded in a surface of low genus; add a bounded number of vortices of bounded depth; then apply a low-rank perturbation. We believe that we have proved that: if $\mathcal{T}$ has sufficiently large order and it does not control an $M\left(K_{n}\right)$ - or $M\left(K_{n}\right)^{*}$-minor, then $\mathcal{T}$ has local structure in $\mathcal{C}$. This result implies the Graph Minors Structure Theorem.

With this result and duality, we can now restrict our attention to tangles that control large cliques.

Excluding $\mathbf{P G}(n, p)$. Let $\mathcal{C}$ be the class of $\mathbb{F}$-representable matroids obtained by low-rank perturbations of Dowling matroids. We conjecture that: If $\mathcal{T}$ controls an $M\left(K_{m}\right)$-minor for a sufficiently large integer $m$ but $\mathcal{T}$ does not control a $\mathrm{PG}(n, p)$-minor, then $\mathcal{T}$ has local structure in $\mathcal{C}$. Notice that vortices seem to vanish once we get off surfaces. This may just be lack of imagination on our behalf, but this is backed-up by several partial results.

Roughly speaking the conjectures above state that: if $M$ is an $\mathbb{F}$ representable matroid with no $P G(n, p)$-minor, then $M$ admits a tree-like decomposition such that each part is either essentially a Dowling matroid or is essentially the dual of a Dowling matroid. For fields of prime order this would give the required constructive structural characterization of its proper minor-closed classes.

Excluding PG $(n, q)$. Suppose that $q$ is not prime and let $\mathcal{C}$ be the class of $\mathbb{F}$-representable matroids obtained from low-rank perturbations of matroids that are representable over subfields of $\mathbb{F}$. We conjecture that: If $\mathcal{T}$ controls a PG( $m, p)$-minor for a sufficiently large integer $m$ but $\mathcal{T}$ does not control a $\operatorname{PG}(n, q)$-minor, then $\mathcal{T}$ has local structure in $\mathcal{C}$.

Finally we can summarize all of the above into a single conjecture. For any proper minor-closed class $\mathcal{M}$ of $\mathbb{F}$-representable matroids, each matroid in $\mathcal{M}$ admits a tree-like decomposition such that each part is either essentially a Dowling matroid, or is essentially the dual of a Dowling matroid, or is essentially represented over a subfield of $\mathbb{F}$.

\section{Back to Rota's Conjecture}

Given that we know that Rota's Conjecture holds for $G F(2), G F(3)$ and $G F(4)$, and that the Well-Quasi-Ordering Conjecture has not been resolved for any field, it is natural to believe that the resolution of Rota's Conjecture is the easier of the the two. But we are gravitating towards the belief that Rota's Conjecture is the more difficult. In [11] we prove that an excluded minor for $G F(q)$-representability of sufficiently large branch width cannot contain a $P G(q+6, q)$-minor. We also know that there are a finite number of such excluded minors for any given branch width. It follows that if 
Rota's Conjecture fails for $G F(q)$, then there must exist excluded minors with arbitrarily large grid minors and no large projective space as a minor. Therefore, a structure theorem for excluding a projective geometry would provide significant structural information about excluded minors.

Assuming that one can prove a structure theorem, how would one go about proving Rota's Conjecture? The proof of Theorem 2.5 provides one possible approach. Consider a planar graph $H$ and finite field $\mathbb{F}$. The results in [12] provide the structural characterization for the class of $\mathbb{F}$-representable matroids with mo $M(H)$-minor, and, by this structural characterization and the results in [14], we see that all but a finite number of the excluded minors for the class of $\mathbb{F}$-representable matroids contain an $M(H)$-minor. The techniques in [14] are quite general and could well extend to prove Rota's Conjecture in full. The idea is to consider the class of matroids $M$ that have an element $e$ such that $M \backslash e$ and $M / e$ are both $\mathbb{F}$-representable, and to show that this class is well-quasi-ordered with respect to taking minors. This approach is however fraught with horrendous technicalities, which we would sooner avoid.

The techniques used in exluding a $P G(q+6, q)$-minor are essentially a generalisation of the techniques used in the resolution of Rota's Conjecture for $G F(4)$ [5] and rely crucially on the fact that a 3-connected matroid with a $P G(q, q)$-minor is uniquely representable. Other than the results in [14], all partial results towards Rota's Conjecture require unique representability. To avoid the techicalities in extending [14], we will need to develop a better understanding the behaviour of inequivalent representations. Unfortunately, as shown in [20], the number of inequivalent representations of 3-connected $G F(q)$-representable matroids is unbounded for all prime powers $q \geq 7$. One way to deal with this is to raise connectivity.

In [13], we prove that for any finite field $\mathbb{F}$, the number of inequivalent representations of 4-connected $G F(q)$-representable matroids is bounded. We also show that, certifying non- $G F(q)$-representability of an $n$-element matroid requires only $O\left(n^{2}\right)$ rank evaluations. From a complexity-theoretic point of view, although not from an aesthetic one, this result is almost as good as Rota's Conjecture. (Rota's Conjecture would imply that we only require a constant number of rank evaluations.)

The results in [13] show promise, but, sadly, they seem inadequate for proving Rota's Conjecture. For Rota's Conjecture, it seems that we need to extend the theory of stabilizers [31] to 4-connected matroids.

The field GF(5) is peculiar with respect to Rota's Conjecture. The results in [31] resolve all issues caused by inequivalent representations. However, Rota's Conjecture remains open for GF(5). The problems encountered when trying to extend the methods of [5] are related to "intertwining". A positive answer to the following conjecture would be of great assistance.

Conjecture 6.1. Let $N$ be a $G F(q)$-representable matroid. Then there is an integer $l$ such that if $M$ is a $G F(q)$-representable matroid with branch 
width $l$ and $M$ contains $N$ as a minor, then there exists $e \in E(M)-E(N)$ such that both $N \backslash e$ and $N /$ e contain $N$ as a minor.

We hope to prove this conjecture as a corollary to the proposed structure theorem for $G F(q)$-representable matroids.

\section{REFERENCES}

[1] Bixby, R. E., On Reid's characterization of the ternary matroids, J. Combin. Theory Ser. B 26 (1979), 174-204.

[2] Dharmatilake, J., A min-max theorem using matroid separations, Matroid Theory (Seattle WA 1995), 333-342, Contemp. Math. 197 Amer. Math. Soc., Providence RI 1996.

[3] Diestel, R., Gorbanov, K., Jensen, T., and Thomassen, K., Highly connected sets and the excluded grid theorem, J. Combin. Theory Ser. B 75 (1999), 61-73.

[4] Dowling, T., A class of geometric lattices based on finite groups. J. Combin. Theory Ser. B 14 (1973), 61-86.

[5] Geelen, J., Gerards, A. M. H., and Kapoor, A., The excluded minors for $G F(4)-$ representable matroids, J. Combin. Theory Ser. B 79 (2000), 247-299.

[6] Geelen, J., Gerards, A. M. H., Robertson, N., and Whittle, G., On the excluded minors of matroids of branch width $k$ J. Combin. Theory Ser. B 88 (2003), 261-265.

[7] Geelen, J., Gerards, A. M. H., Robertson, N., and Whittle, G., Obstructions to branch decompositions in matroids. To appear in J. Combin. Theory Ser. B.

[8] Geelen, J., Gerards, A. M. H., and Whittle, G., Branch-width and well-quasi-ordering in matroids and graphs, J. Combin. Theory Ser. B, 84 (2002), 270-290.

[9] Geelen, J., Gerards, A. M. H., and Whittle, G., Disjoint cocircuits in matroids with large rank, J. Combin. Theory Ser. B 87 (2003), 270-279.

[10] Geelen, J., Gerards, B., and Whittle, G., Tangles, tree decompositions and grids in matroids, preprint.

[11] Geelen, J., Gerards, B., and Whittle, G., On Rota's Conjecture and excluded minors containing large projective geometries, to appear in J. Combin. Theory Ser. B.

[12] Geelen, J., Gerards, B., and Whittle, G., Excluding a planar graph from $G F(q)$ representable matroids, submitted.

[13] Geelen, J., Gerards, B., and Whittle, G., Inequivalent representations of matroids I: An overview, in preparation.

[14] Geelen, J., and Whittle, G., Branch width and Rota's conjecture, J. Combin. Theory Ser. B, 86 (2002), 315-330.

[15] Geelen, J., and Whittle, G., Cliques in dense $G F(q)$-representable matroids, J. Combin. Theory Ser. B, 87 (2003), 264-269.

[16] Hliněný, P., Branch-width, parse trees, and monadic second-order logic for matroids, preprint. Extended abstract in: STACS 2003.

[17] Hliněný, P., and Whittle, G., Matroid tree width, to appear in European Journal of Combinatorics.

[18] Johnson, T., Robertson N., and Seymour P. D., Connectivity in binary matroids. Handwritten manuscript.

[19] Lazarson, T., The representation problem for independence functions. J. London Math. Soc. 33 (1958), 21-25.

[20] Oxley, J. G., Vertigan, D. L. and Whittle, G. P., On inequivalent representations of matroids over finite fields, J. Combin. Theory Ser. B 67 (1996), 325-343.

[21] Robertson, N. and Seymour, P. D., Graph Minors. IV. Tree-width and well-quasiordering. J. Combin. Theory Ser. B 48 (1990), 227-254.

[22] Robertson, N. and Seymour, P. D., Graph minors. V. Excluding a planar graph, J. Combin. Theory Ser. B 41 (1986), 92-114. 
[23] Robertson, N. and Seymour, P. D., Graph minors. X. Obstructions to tree decomposition, J. Combin. Theory Ser. B 52 (1991), 153-190.

[24] Robertson, N. and Seymour, P. D., Graph Minors. X111. The disjoint paths problem, J. Combin. Theory Ser. B 63 (1995), 65-110.

[25] Robertson, N. and Seymour, P. D., Graph Minors. XX. Wagner's Conjecture, J. Combin. Theory Ser. B 92 (2004), 325-357.

[26] Robertson, N., Seymour, P. D., and Thomas, R., Quickly excluding a planar graph, J. Combin. Theory Ser. B 62 (1994), 323-348.

[27] Rota, G. -C., Combinatorial theory, old and new. In Proc. Internat. Cong. Math. (Nice, Sept. 1970), pp. 229-233. Gauthier-Chichester (1970).

[28] Seymour, P. D., Matroid representation over GF(3), J. Combin. Theory Ser. B 26 (1979), 159-173.

[29] Tutte, W. T., A homotopy theorem for matroids, I, II, Trans. Amer. Math. Soc. 88 (1958), 144-174.

[30] Tutte, W. T., Menger's theorem for matroids, Journal of Research of the National Bureau of Standards - B. Mathematics and Mathematical Physics, 69B (1965), 49-53.

[31] Whittle, G. P., Stabilizers of classes of representable matroids, J. Combin. Theory Ser. B 77 (1999), 39-72.

[32] Zaslavsky, T., Biased graphs. II. The three matroids, J. Combin. Theory Ser. B 51 (1991), 46-72.

[33] Zaslavsky, T., A mathematical bibliography of signed and gain graphs and allied areas. Manuscript prepared with Marge Pratt. Electron. J. Combin. 5 (1998), Dynamic Surveys 8, 124 pp. (electronic).

Department OF COMBINATORICS AND Optimization, University OF WATERloo, WATERloO, ONTARIO, CANADA

E-mail address: jfgeelen@uwaterloo.ca

CWi, Amsterdam, The Netherlands., Department of Mathematics and Computer Science, Eindhoven University of Technology, Eindhoven, The NetherLANDS

E-mail address: bert.gerards@cwi.nl

School of Mathematical and Computing Sciences, Victoria University, Wellington, NeW ZeAland

E-mail address: geoff.whittle@vuw.ac.nz 